\title{
Adjuvantes na deriva de 2,4-D + glyphosate em condições de campo
}

\author{
Adjuvants on spray drift of 2,4-D + glyphosate in field conditions
}

\begin{abstract}
Augusto Guerreiro Fontoura Costa ${ }^{\mathrm{I}}$ Edivaldo Domingues Velini ${ }^{\mathrm{II}}$ Caio Vitagliano Santi Rossi ${ }^{\mathrm{II}}$

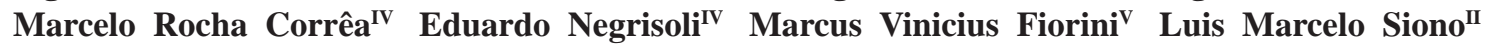

RESUMO

O objetivo do trabalho foi avaliar o efeito de adjuvantes sobre a deriva em aplicações da mistura de 2,4-D + glyphosate. $O$ experimento foi conduzido em condições de campo em delineamento inteiramente casualizado. Os tratamentos corresponderam às soluções com a mistura dos herbicidas 2,4-D

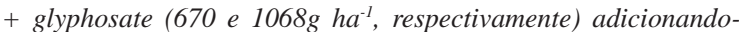
se os adjuvantes ( $\left.v v^{-1}\right)$ : óleo mineral $(0,5 \%)$; agente antideriva $(0,09 \%)$; espalhante adesivo A $(0,1 \%) ;$ fertilizante líquido (0,05\%); espalhante adesivo $B(0,25 \%)$; e somente os herbicidas (testemunha). Foram utilizados para determinação de deriva fios de náilon externos à área de aplicação (1, 5, 10, 20, 50, 100 e 200m de distância) com quatro repetições e seis cilindros de espuma posicionados sobre a barra do pulverizador para coleta de gotas sujeitas à deriva. As aplicações foram realizadas simultaneamente, utilizando-se um sal traçador específico em cada solução de aplicação para quantificar os depósitos por meio de espectrofotômetro. Não foi possível verificar efeito dos adjuvantes sobre a deriva nas diferentes distâncias da área de aplicação. Com base nas gotas coletadas acima da barra de pulverização, constatou-se que a suscetibilidade à deriva foi menor com o óleo mineral e o agente antideriva. $O$ risco de deriva foi maior com o fertilizante líquido e o espalhante adesivo $B$.

Palavras-chave: herbicida, coletor de deriva, pulverizador de barra, aditivo.

\section{ABSTRACT}

The aim of the research was to evaluate the effect of adjuvants on the spray drift applications from mixture of 2,4$D+$ glyphosate. The trial was carried out in field conditions in a completely randomized design. The treatments corresponded to solutions containing mixture of the herbicides 2,4-D + glyphosate (670 and $1068 \mathrm{~g} \mathrm{ha}^{-1}$, respectively) adding the adjuvants $\left(v \mathrm{v}^{-1}\right)$ : mineral oil (0.5\%); anti-drift agent (0.09\%); spreader-sticker A (0.1\%); liquid fertilizer (0.05\%); spreader-sticker B (0.25\%); and only herbicides without adjuvantes (control). Nylon strings were used to drift determination outside the application area $(1,5,10$, 20, 50, 100 and $200 \mathrm{~m}$ away) with 4 replications and six foam cylinders placed on the boom of the sprayer were used to collect the droplets subject to drift. The applications were performed simultaneously, using a specific salt tracer for each spray solution to quantify the deposits by spectrophotometer. It was not possible to verify effect of the adjuvants on drift at different distances of the application area. Based on droplets collected above the boom spray, it was found that susceptibility to drift was lower with the mineral oil and the anti-drift agent. The drift risk was higher with the liquid fertilizer and the spreader-sticker $B$.

Key words: herbicide, drift collector, boom sprayer, additive.

\section{INTRODUÇÃO}

A deriva pode ser definida como parte da pulverização agrícola desviada para fora da área-alvo pela ação do vento (MILLER, 1993), constituindo-se uma das principais causas de perdas de herbicidas. Em relação aos problemas decorrentes de deriva, o herbicida 2,4-D tem se destacado por causa da elevada toxicidade às culturas sensíveis e pelo intensivo uso, com destaque para as aplicações de manejo no sistema plantio direto no Brasil.

\footnotetext{
IEmpresa Brasileira de Pesquisa Agropecuária, Embrapa Algodão, 58428-095, Campina Grande, PB, Brasil. E-mail: augusto.costa@embrapa.br. Autor para correspondência.

IINúcleo de Pesquisas Avançadas em Matologia (Nupam), Faculdade de Ciências Agronômica (FCA), Universidade Estadual Paulista (UNESP), Botucatu, SP, Brasil.

IIIDow AgroSciences Industrial Ltda, Uberlândia, MG, Brasil.

IVTechfield Assessoria e Consultoria Agrícola Ltda, Botucatu, SP, Brasil.

vDow AgroSciences Industrial Ltda, São Paulo, SP, Brasil. 
Embora nas operações de manejo o 2,4-D é comumente associado ao glyphosate, objetivando maior número de espécies a serem controladas, os maiores riscos de intoxicação têm sido associados ao primeiro em decorrência do efeito em doses extremamente baixas. A dose pode corresponder, por exemplo, a $1 \%$ do total aplicado $\left(6,72 \mathrm{~g} \mathrm{ha}^{-1}\right) \mathrm{em}$ culturas sensíveis, como o algodoeiro e uva na fase de florescimento, para as quais foram verificadas, respectivamente, diminuições de $62 \%$ e $85 \%$ na produtividade (CONSTANTIN et al., 2007; OLIVEIRA JÚNIOR et al., 2007).

$\mathrm{O}$ uso predominante do glyphosate para culturas resistentes tem representado múltiplas oportunidades de ocorrência de deriva (JOHNSON et al., 2006), além de ser um dos herbicidas mais utilizados no mundo (GHISI \& CESTARI, 2013). Sua toxicidade também foi relatada para diversos cultivos. YAMASHITA \& GUIMARÃES (2006) verificaram redução de $40 \%$ na produtividade de algodão a partir da menor dose avaliada $\left(180 \mathrm{~g} \mathrm{ha}^{-1}\right)$ no estádio de 4 folhas. TUFFI SANTOS et al. (2006) observaram diminuição no crescimento de plantas de eucalipto submetidas a $172,8 \mathrm{~g} \mathrm{ha}^{-1}$ de glyphosate aos 45 dias após o transplante. FIGUEREDO et al. (2007) apresentaram reduções na altura de plantas, número de flores, cachos florais e frutos de tomateiro a partir de $189 \mathrm{~g} \mathrm{ha}^{-1}$ ( $15 \%$ da dose do herbicida) em deriva simulada, ocorrida aos 70 dias após o transplante.

Portanto, em virtude do uso frequente de glyphosate e 2,4-D e do risco de fitointoxicação de ambos a várias espécies vegetais, fatores como condições climáticas, distância de culturas sensíveis da área-alvo e técnicas de aplicação devem ser sempre considerados e avaliados, com o objetivo de minimizar o risco ou a magnitude do processo de deriva.

Entre as técnicas de aplicação a serem definidas, adjuvantes adicionados para modificar as características da solução de aplicação podem ser utilizados (MOREIRA JUNIOR \& ANTUNIASSI, 2010). FELSOT et al. (2010) ainda mencionam que os adjuvantes podem influenciar fatores que são determinantes para a deriva, como o tamanho das gotas pulverizadas. Como exemplo, JOHNSON et al. (2006) obtiveram redução de deriva com adjuvante voltado para esse fim em condições de campo. COSTA et al. (2008) verificaram aumento no tamanho das gotas e redução de deriva em condições controladas, com adição de óleo vegetal a $1 \%\left(\mathrm{~V} \mathrm{v}^{-1}\right)$ na mistura de 2,4-D com glyphosate.

Apesar dos adjuvantes serem considerados entre tecnologias que podem influenciar a deriva de herbicidas, ainda são escassos os estudos disponíveis a esse respeito no Brasil, principalmente em condições de campo. Em razão do exposto, o objetivo deste trabalho foi avaliar o efeito de adjuvantes sobre a deriva em aplicações de 2,4-D + glyphosate.

\section{MATERIAL E MÉTODOS}

O estudo foi realizado no Município de Bocaina-SP, em área de pousio anteriormente cultivada com cana-de-açúcar. A área utilizada para aplicação foi de 1 ha com formato retangular, cujos lados corresponderam a 50 e $200 \mathrm{~m}$.

Os adjuvantes avaliados e suas respectivas composições foram: óleo mineral (mistura de hidrocarbonetos parafínicos, ciclo parafínicos, aromáticos e insaturados, provenientes da destilação do petróleo a $761 \mathrm{~g} \mathrm{~L}^{-1}$ ), agente antideriva [(hidroxipropil guar, alquil poliglicosídeo a 75\% (p $\left.\mathrm{p}^{-1}\right)$ ]; espalhante adesivo A (copolímero de poliéter e silicone a $\left.1.000 \mathrm{~g} \mathrm{~L}^{-1}\right)$; fertilizante líquido $\left(\mathrm{Ne}_{2} \mathrm{O}_{5}\right.$ a 36 e $204 \mathrm{~g} \mathrm{~L}^{-1}$, respectivamente) e espalhante adesivo B (alcoofenóis+óxido de eteno+sulfatos orgânicos a $\left.466 \mathrm{~g} \mathrm{~L}^{-1}\right)$. Os herbicidas 2,4-D e glyphosate foram utilizados nas doses de 670 e $1068 \mathrm{~g}$ e. a. ha ${ }^{-1}$, respectivamente. Os tratamentos foram compostos por soluções de aplicação contendo a mistura dos herbicidas adicionados ou não dos adjuvantes nas seguintes concentrações $\left(\mathrm{V} \mathrm{V}^{-1}\right)$ : testemunha (herbicidas sem adjuvante); herbicidas + óleo mineral a $0,5 \%$; herbicidas + agente antideriva a $0,09 \%$; herbicidas + espalhante adesivo A a $0,1 \%$; herbicidas + fertilizante a $0,05 \%$ e herbicidas + espalhante adesivo B a $0,25 \%$.

Para que as aplicações de todos os tratamentos fossem simultâneas, foi utilizado um pulverizador pressurizado com $\mathrm{CO}_{2}$ desenvolvido especificamente para este tipo de estudo (COSTA et al., 2012), com compartimento de calda, registros, válvulas reguladoras de pressão e seções de barra individualizadas. $\mathrm{O}$ aparelho foi acoplado a um pulverizador com barra de $11 \mathrm{~m}$ (Jacto PJ-400). Por serem simultâneas as aplicações, utilizou-se um sal traçador na concentração de $20 \mathrm{~g} \mathrm{~L}^{-1}$ específico em cada solução de aplicação, sendo: $\mathrm{MnSO}_{4}, \mathrm{NaCl}$, $\mathrm{ZnCl}, \mathrm{MgCl}, \mathrm{CaCl}, \mathrm{KCl}$ para a testemunha (sem adjuvantes), tratamentos contendo óleo, agente antideriva, espalhante adesivo A, fertilizante e espalhante adesivo B, respectivamente. As soluções de aplicação foram armazenadas em tanques de pulverização individuais com capacidade de $11 \mathrm{~L}$ acoplados ao trator. A velocidade de deslocamento foi de $4,3 \mathrm{~km} \mathrm{~h}^{-1}$, sendo utilizados quatro bicos para 
cada tratamento, espaçados a $50 \mathrm{~cm}$, com as pontas de pulverização de jato plano XR 11002, operando a $300 \mathrm{kPa}$ de pressão e resultando em pulverização de gotas de tamanho médio, com diâmetro mediano volumétrico de $220 \mu \mathrm{m}$ (ANTUNIASSI \& BAIO, 2008). Para cada aplicação, foi retirada uma amostra de calda para permitir posteriormente a quantificação exata do traçador em laboratório.

Para coletar as gotas de menor tamanho que não atingissem diretamente o alvo, ficando suspensas na região da altura da barra de pulverização e, consequentemente, sob risco de deriva, foram utilizados cilindros de espuma com $2400 \mathrm{~mm}$ de comprimento por $100 \mathrm{~mm}$ de diâmetro, conforme método proposto por COSTA et al. (2012). Para tanto, seis unidades coletoras foram distribuídas e fixadas sobre a barra do pulverizador, com auxílio de armações de canos de PVC. Um cilindro foi posicionado acima de cada conjunto de pontas de pulverização, de modo a priorizar a coleta das gotas de cada tratamento.

Os alvos utilizados como coletores de deriva foram fios de náilon com dimensão de $2 \mathrm{~mm}$ de diâmetro e $1200 \mathrm{~mm}$ de comprimento, os quais foram posicionados verticalmente em hastes metálicas fixadas a $15 \mathrm{~cm}$ de altura em relação ao solo nas distâncias de 1, 5, 10, 20, 50, 100 e 200m da área de aplicação. Perpendicular e no centro de cada lado da área a ser aplicada foi posicionada uma fileira de coletores nas referidas distâncias, totalizando quatro repetições em delineamento inteiramente casualizado.

Foram realizadas dez aplicações em área total, a cada $50 \mathrm{~min}$, ao longo de um dia, iniciadas às $9 \mathrm{~h} 35 \mathrm{~min}$ e finalizadas às $17 \mathrm{~h} 05 \mathrm{~min}$, permitindo que os dados obtidos fossem resultantes do somatório de depósitos para favorecer a posterior detecção e quantificação dos traçadores.

Durante as aplicações, a velocidade do vento, a temperatura e a umidade relativa do ar foram registradas por meio de uma estação meteorológica portátil instalada na área experimental, cujos valores mínimo, médio e máximo corresponderam, respectivamente, a: 0,$0 ; 9,5$ e $20,2 \mathrm{~km} \mathrm{~h}^{-1} ; 17,7$; 27,9 e $32,6^{\circ} \mathrm{C} ; 28,0 ; 39,5$ e $71,0 \%$. De acordo com as condições climáticas adequadas para aplicações de defensivos agrícolas por meio de pulverização, ANDEF (2004), a velocidade média do vento ao longo do dia se encontrava próxima do limite de $10 \mathrm{~km} \mathrm{~h}^{-1}$ e chegou a atingir o valor máximo de $20 \mathrm{~km}$ $\mathrm{h}^{-1}$, portanto, favorável à comparação de tecnologias que pudessem influenciar na magnitude do processo de deriva. Os somatórios dos depósitos de deriva nos coletores permitiram obter dados resultantes do efeito da variação da condição climática ao longo do dia utilizado para a avaliação.

Ao final das dez aplicações, os fios de náilon e cilindros de espuma foram recolhidos e armazenados em frascos de PVC e sacos de polietileno, respectivamente. Posteriormente, em laboratório, cada uma das unidades coletoras foi lavada com água destilada, com volumes de 25 e 500mL para cada fio de náilon e cilindro de espuma, respectivamente, e as soluções de lavagem analisadas em espectrofotômetro de plasma. As concentrações dos traçadores foram obtidas considerando-se o coeficiente de extração dos sais utilizados. Posteriormente, foram corrigidas, padronizando-se o volume de calda para 200 L ha-1 e transformadas em volume de calda depositado por unidade de área da superfície coletora $\left(\mu \mathrm{L} \mathrm{cm}^{-2}\right)$. Para os coletores posicionados acima da barra de pulverização, a comparação entre os tratamentos foi realizada de maneira direta com as quantidades de depósitos obtidas. Para os alvos de fios de náilon, os dados foram submetidos à análise de variância pelo teste $\mathrm{F}$ e as médias comparadas pelo teste $\mathrm{t}$ a $5 \%$ de probabilidade.

\section{RESULTADOS E DISCUSSÃO}

Na tabela 1, estão apresentados os depósitos de calda das soluções de aplicação de herbicida coletados fora da área-alvo (deriva) nas diferentes distâncias de coleta. Todavia, quando consideradas somente as diferenças numéricas entre as médias apresentadas na tabela 1, é possível verificar que ocorreu tendência de menor perda da pulverização para o agente antideriva, especialmente nas distâncias de 1 a $10 \mathrm{~m}$, para as quais as diferenças entre as soluções de aplicação foram maiores. Entretanto, principalmente na distância de $1 \mathrm{~m}$, pode ser observada tendência de maior deriva para o espalhante adesivo B. CELEN (2010), ao avaliar o efeito de adjuvantes sobre a deriva, constatou maiores diferenças entre os tratamentos também nas distâncias mais próximas do alvo (até $3 \mathrm{~m}$ ), entretanto, os menores depósitos ocorreram a $15 \mathrm{~m}$ de distância por causa da adição de um adjuvante antideriva, composto pela mistura de sulfato de diamônio, polímero e antiespumante.

Em geral, podem-se constatar quantidades decrescentes de deriva em função do aumento da distância do coletor em relação à área-alvo. Resultados de campo encontrados na literatura também demonstram que quanto maior a distância de coleta, menor a deriva detectada (NUYTTENS et al., 2006). CARLSEN et al. reportam que faixas de terreno sem pulverização nas bordaduras da áreaalvo (buffer zones) têm sido avaliadas como proteção 
Tabela 1 - Depósitos de deriva de calda $\left(\mu \mathrm{L} \mathrm{cm}^{-2}\right)$ detectados nas posições de coleta fora da área de aplicação, em função da adição de adjuvantes à mistura do 2,4-D + glyphosate.

\begin{tabular}{|c|c|c|c|c|c|c|c|}
\hline Tratamento & $1 \mathrm{~m}$ & $5 \mathrm{~m}$ & $10 \mathrm{~m}$ & $20 \mathrm{~m}$ & $50 \mathrm{~m}$ & $100 \mathrm{~m}$ & $200 \mathrm{~m}$ \\
\hline Testemunha & 14,31 & 9,40 & 6,28 & 2,39 & 1,21 & 0,14 & 0,05 \\
\hline Óleo mineral & 100,01 & 40,35 & 18,32 & 24,10 & 10,79 & 4,99 & 3,09 \\
\hline Ag. antideriva & 7,48 & 5,62 & 3,92 & 1,99 & 1,13 & 0,10 & 0,49 \\
\hline Esp. adesivo A & 41,65 & 37,87 & 24,92 & 14,78 & 7,63 & 1,01 & 1,85 \\
\hline Fertilizante & 8,89 & 5,55 & 7,82 & 10,57 & 2,01 & 4,70 & 13,99 \\
\hline Esp. adesivo B & 140,23 & 39,50 & 17,03 & 7,61 & 6,49 & 4,68 & 7,18 \\
\hline $\mathrm{F}$ & $1,03^{\mathrm{ns}}$ & $1,51^{\mathrm{ns}}$ & $0,91^{\mathrm{ns}}$ & $1,03^{\mathrm{ns}}$ & $1,05^{\mathrm{ns}}$ & $0,98^{\mathrm{ns}}$ & $1,56^{\mathrm{ns}}$ \\
\hline $\mathrm{CV}(\%)$ & 210,65 & 125,75 & 133,05 & 161,42 & 160,88 & 187,51 & 192,13 \\
\hline
\end{tabular}

* significativo a $5 \%$ de probabilidade; ${ }^{\text {NS }}$ não significativo.

às áreas vizinhas. Apesar de não existir consenso entre os pesquisadores, se essas faixas protegem suficientemente os arredores, distâncias entre 3 e $24 \mathrm{~m}$ têm sido mencionadas como apropriadas. Entretanto, os próprios autores detectaram deriva de herbicidas até $150 \mathrm{~m}$ de distância em aplicações terrestres. HEWITT et al. (2009) verificaram que áreas que recebem a aplicação aérea de glyphosate deveriam estar entre 50 e $120 \mathrm{~m}$ de distância de culturas sensíveis. Em geral, pesquisas a campo, como o presente estudo, nas quais se quantificam os depósitos de calda fora da áreaalvo, têm sido mais utilizadas para avaliar o efeito de fatores que possam contribuir para redução da deriva. A faixa ou distância mínima de segurança da área de aplicação tem sido preferencialmente determinada a partir de estudos em condições controladas, como em túnel de vento e/ou a partir de modelos de previsão.

Ainda pela tabela 1 , não é possível constatar diferenças entre os tratamentos, independente da distância, muito provavelmente em virtude da elevada variabilidade dos dados. Apesar de alvos cilíndricos de $2 \mathrm{~mm}$ de espessura possuírem características desejáveis para coleta de deriva (dimensão reduzida, área definida, fracionável e facilidade no manuseio), a dinâmica combinada de evaporação e sedimentação das gotas pode resultar em baixa eficiência de captura por meio desses coletores passivos. Outra dificuldade pode ser a dispersão da nuvem pulverizada acima de uma altura viável para coleta das gotas (MILLER, 1993). Como exemplo de variabilidade nos valores de deriva, COSTA et al. (2005) obtiveram coeficientes de variação de 359 e $560 \%$ em fios de náilon posicionados até $16 \mathrm{~m}$ de distância da área de aplicação. DONKERSLEY \& NUYTTENS (2011) também mencionam que as condições ambientais em experimentos de campo podem causar variabilidade nas medições de deriva, destacando a temperatura e umidade interferindo na taxa de evaporação e a estabilidade atmosférica das gotas, bem como ventos acima de $18 \mathrm{~km} \mathrm{~h}^{-1}$ sendo prejudiciais para esse tipo de estudo.

Para os coletores posicionados acima da barra de pulverização (Figura 1), as quantidades de calda depositadas, resultantes de gotas suspensas sujeitas à deriva, tenderam a ser maiores para a calda contendo o espalhante adesivo B, seguido do fertilizante líquido, correspondendo, respectivamente, a 124 e 94\% mais depósito em relação ao tratamento sem adjuvantes. MOREIRA JUNIOR \& ANTUNIASSI (2010) verificaram maiores valores de deriva em condições de túnel de vento para um surfactante composto de lauril éter sulfato sódico adicionado à água a $0,2 \%\left(\mathrm{v} \mathrm{v}^{-1}\right)$.

$\mathrm{O}$ agente anti-deriva e o óleo mineral resultaram em tendências de menores valores de deriva, inferiores à solução testemunha (Figura 1). A adição do espalhante adesivo A resultou em tendência de valor intermediário. JOHNSON et al. (2006) também observaram diminuição de deriva de glyphosate com a adição de um adjuvante antideriva.

Ao considerar a relação do aumento do tamanho das gotas com a redução de deriva, HOLLOWAY et al. (2000) verificaram incremento no diâmetro das gotas (DMV) com adjuvantes a base de silicone, óleo mineral ou vegetal. CUNHA et al. (2003) constataram que óleo vegetal na calda de pulverização aumentou o diâmetro das gotas e reduziu a deriva em aplicações de fungicida na cultura do feijoeiro. Entretanto, CUNHA et al. (2010) não verificaram alteração da porcentagem do volume de gotas menores que $100 \mu \mathrm{m}$ produzidas por pontas de pulverização de jato plano defletor, sem e com indução de ar, combinadas ao uso de diferentes adjuvantes, indicados como espalhantes adesivos, entre os quais também constava um produto a base de óleo vegetal. As variações entre esses resultados podem ser explicadas pelo efeito da interação das 


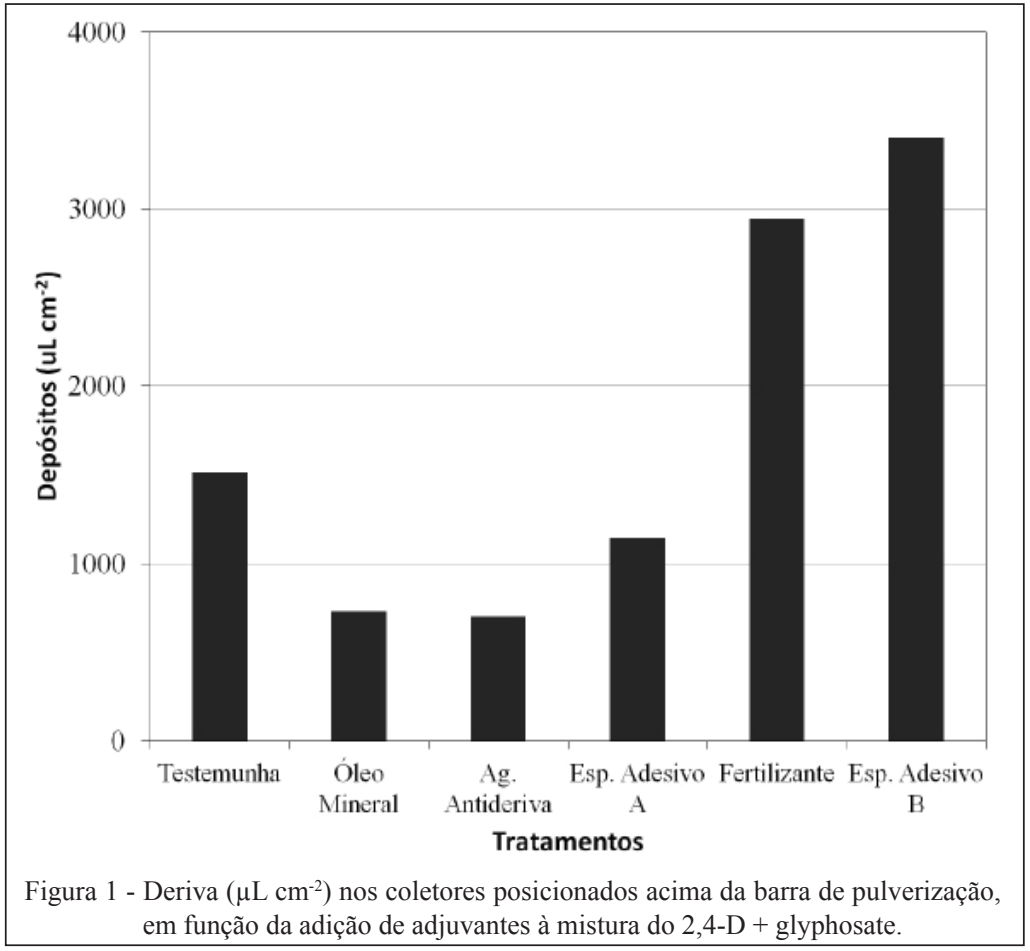

distintas características das tecnologias envolvidas nesse tipo de estudo, pois o risco de deriva, além de ser influenciado pela ponta de pulverização, é afetado pela formulação do agrotóxico e os adjuvantes (HILZ \& VERMEER, 2013).

Especificamente para a aplicação de 2,4$\mathrm{D}+$ glyphosate, utilizando a ponta de pulverização XR11002 a 300kPa, COSTA et al. (2008) constataram redução da deriva destacada com o uso de um adjuvante a base de óleo vegetal, na dose de $1,0 \%$ $(\mathrm{v} / \mathrm{v})$ em túnel de vento, relacionando essa diminuição com o aumento no tamanho das gotas avaliadas. A redução de deriva também foi obtida com um adjuvante a base de silicone e um óleo mineral (25 e $44 \%$, respectivamente), entretanto, o efeito destes foi evidente somente quando adicionado à água, sem a presença dos herbicidas. Sendo assim, os autores mencionam a importância das diferenças entre as formulações, pois adjuvantes também são utilizados para constituir os agrotóxicos comercializados, o que poderia explicar a atenuação do efeito desses dois produtos na presença dos herbicidas.

No presente estudo, de maneira semelhante, também foi constatada tendência de menor deriva de 2,4-D + glyphosate, devido à adição de óleo mineral e um espalhante adesivo a base de silicone. Sendo assim, a seleção adequada de adjuvantes pode ser utilizada como estratégia para redução do risco de deriva dessa mistura de herbicidas.

\section{CONCLUSÃO}

Para a aplicação dos herbicidas 2,4-D + glyphosate, foi possível concluir que a suscetibilidade à deriva foi menor com a adição de óleo mineral e o agente antideriva. Houve maior risco de deriva com a adição do fertilizante líquido e o espalhante adesivo B.

\section{AGRADECIMENTOS}

À Dow AgroSciences Industrial Ltda pelo suporte financeiro e à Tonon Bionergia S. A. pelo apoio e área cedida ao experimento.

\section{REFERÊNCIAS}

ANTUNIASSI, U.R; BAIO, F.H.R. Tecnologia de aplicação de defensivos. In: VARGAS, L.; ROMAN, E.S. Manual de manejo e controle de plantas daninhas. Passo Fundo: Embrapa Trigo, 2008. p.173-212.

ASSOCIAÇÃO NACIONAL DE DEFESA VEGETAL (ANDEF). Manual de tecnologia de aplicação de produtos fitossanitários. Campinas: Linea Creativa, 2004. 50p.

CARLSEN, S.C.K et al. Drift of 10 herbicides after tractor spray application. 2. Primary drift (droplet drift). Chemosphere, v.64, n.5, p.778-786, 2006. Disponível em: <http://www.sciencedirect. com/science/article/pii/S0045653505012713>. Acesso em: 4 jun. 2013. doi:10.1016/j.chemosphere.2005.10.060.

CELEN, I.H. The effect of spray mix adjuvants on spray drift. Bulgarian Journal of Agricultural Science, v.16, n.1, p.105-110, 2010. Disponível em: <http://www.agrojournal.org/16/01-15. htm>. Acesso em: 27 ago. 2012. 
CONSTANTIN, J. et al. Efeito de subdoses de 2,4-D na produtividade do algodão e suscetibilidade da cultura em função de seu estádio de desenvolvimento. Engenharia Agrícola, v.27, n. esp., p.24-29, 2007. Disponível em: <http://www.scielo.br/pdf/ eagri/v27nspe/04.pdf>. Acesso em: 27 ago. 2012. doi:10.1590/ S0100-69162007000200004.

COSTA, A.G.F. et al. Determinação da deriva de 2,4-D e glyphosate com diferentes pontas de pulverização e adjuvantes. In: CONGRESO de la ASOCIACIÓN LATINO-AMERICANA DE MALEZAS, 28., 2008, Ouro Preto, MG. Anais... Sete Lagoas: SBCPD / Embrapa Milho e Sorgo, 2008. 1 CD.

COSTA, A.G.F. et al. Efeito de pressões e pontas de pulverização na deriva de 2,4-D + glyphosate em condições de campo. Revista Brasileira de Herbicidas, v.11, n.1, p.62-70, 2012. Disponível em: $<$ http://www.rbherbicidas.com.br/index.php/rbh/article/view/143>. Acesso em: 27 ago. 2012. doi: 10.7824/rbh.v11i1.143.

COSTA, A.G.F. et al. Methods to estimate losses in preemergence herbicide sprays. Bulletin of Environmental Contamination and Toxicology, v.74, n.8, p.8-15, 2005. Disponível em: <http:// link.springer.com/article/10.1007/s00128-004-0541-7>. Acesso em: 4 jun. 2013. doi: 10.1007/s00128-004-0541-7.

CUNHA, J.P.A.R. et al. Avaliação de estratégias para redução da deriva de agrotóxicos em pulverizações hidráulicas. Planta Daninha, v.21, n.2, p.325-332, 2003. Disponível em: <http:// www.scielo.br/pdf/pd/v21n2/a19v21n2.pdf $>$. Acesso em: 27 ago. 2012. doi: 10.1590/S0100-83582003000200019.

CUNHA, J.P.A.R. et al. Espectro de gotas de pulverização com adjuvantes de uso agrícola. Planta Daninha, v.28, n. esp, p.1153-1158, 2010. Disponível em: <http://www.scielo.br/pdf/ pd/v28nspe/a23v28nspe.pdf >. Acesso em: 27 ago. 2012. doi: 10.1590/S0100-83582010000500023.

DONKERSLEY, P.; NUYTTENS, D. A meta analysis of spray drift sampling. Crop Protection, v.30, n.7, p.931-936, 2011 Disponível em: <http://www.sciencedirect.com/science/article/pii/ S0261219411001098>. Acesso em: 4 jun. 2013. doi:10.1016/j. cropro.2011.03.020.

FELSOT, A.S. et al. Agrochemical spray drift; an assessment and mitigation - A review. Journal of Environmental Science and Health Part B - Pesticide Food Contaminants and Agricultural Wastes, v.46, n.1, p.1-23, 2010. Disponível em: <http://www.ncbi. nlm.nih.gov/pubmed/20981606>. Acesso em: 27 ago. 2012. doi: 10.1080/03601234.2010.515161.

FIGUEREDO, S.S. et al. Influência de doses reduzidas do glyphosate no tomateiro (Lycopersicon esculentum Mill.). Planta Daninha, v.25, n.4, p.849-857, 2007. Disponível em: <http:// www.scielo.br/pdf/pd/v25n4/a22v25n4.pdf>. Acesso em: 27 ago. 2012. doi: 10.1590/S0100-83582007000400022.

GHISI, N.C.; CESTARI, M.M. Genotoxic effects of the herbicide Roundup ${ }^{\circledR}$ in the fish Corydoras paleatus (Jenyns 1842) after short-term, environmentally low concentration exposure. Environmental Monitoring Assessement, v.185, n.4, p.32013207, 2013. Disponível em: <http://www.ncbi.nlm.nih.gov/ pubmed/22821326>. Acesso em: 03 jun. 2013. doi: 10.1007/ s10661-012-2783-x.

HEWITT, A.J. et al. Spray droplet size, drift potential, and risks to nontarget organisms from aerially applied glyphosate for coca control in Colombia. Journal of Toxicology and Environmental Health Part A, v.72, n.15-16, p.921-929, 2009. Disponível em: $<$ http://www.ncbi.nlm.nih.gov/pubmed/19672761>. Acesso em: 03 jun. 2013. doi: 10.1080/15287390902929667.

HILZ, E.; VERMEER, A. Spray drift review: the extent to which a formulation can contribute to spray drift reduction. Crop Protection, v.44., p.75-83, 2013. Disponível em: <http://www. sciencedirect.com/science/article/pii/S0261219412003006>. Acesso em: 06 jun. 2013. doi: 10.1016/j.cropro.2012.10.020.

HOLLOWAY, P.J. et al. Effects of some agricultural tank-mix adjuvants on the deposition efficiency of aqueous sprays on foliage. Crop Protecion, v.19, p.29-37, 2000. Disponível em: <http:// www.sciencedirect.com/science/article/pii/S0261219499000794>. Acesso em: 27 ago. 2012. doi:10.1016/S0261-2194(99)00079-4.

JOHNSON, A.K. et al. Glyphosate spray drift management with drift reducing nozzles and adjuvants. Weed Technology, v.20, p.893-897, 2006. Disponível em: <http://www.bioone. org/doi/abs/10.1614/WT-05-162.1>. Acesso em: 27 ago. 2012. doi:10.1614/WT-05-162.1

OLIVEIRA JÚNIOR et al. Efeito de subdoses de 2,4-D na produtividade de uva itália e suscetibilidade da cultura em função de seu estádio de desenvolvimento. Engenharia Agrícola, v.27, n. esp., p.35-40, 2007. Disponível em: <http://www.scielo.br/pdf/ eagri/v27nspe/06.pdf $>$. Acesso em: 18 nov. 2013. doi: 10.1590/ S0100-69162007000200006

MILLER, P.C.H. Spray drift and its measurement. In: MATTHEWS, G.A.; HISLOP, E.C. Application technology for crop protection. Wallingford: CAB International, 1993. p.101-122.

MOREIRA JUNIOR, O.; ANTUNIASSI, U.R. Construção e validação de um túnel de vento para ensaios da estimativa da deriva em pulverizações agrícolas. Energia na Agricultura, v.25, n.3, p.118-136, 2010. Disponível em: <http://200.145.140.50/ index.php/energia/article/view/71>. Acesso em: 27 ago. 2012.

NUYTTENS, D. et al. Experimental study of factors influencing the risk of drift from field sprayers, Part 2: Spray application technique. Aspects of Applied Biology, v.77, p.331-339, 2006.

TUFFI SANTOS, L.D. et al. Intoxicação de espécies de eucalipto submetidas à deriva de glyphosate. Planta Daninha, v.24, n.2, p.359-364, 2006. Disponível em: <http://www.scielo.br/pdf/pd/ v24n2/30461.pdf $>$. Acesso em: 06 jun. 2013. doi: 10.1590/s010083582006000200020 .

YAMASHITA, O.M; GUIMARÃES, S.C. Deriva simulada de glyphosate em algodoeiro: efeito de dose, cultivar e estádio de desenvolvimento. Planta Daninha, v.24, n.4, p.821-826, 2006. Disponível em: <http://www.scielo.br/pdf/pd/v24n4/ a24v24n4.pdf>. Acesso em: 27 ago. 2012. doi: 10.1590/S010083582006000400024 